\title{
CONSERVING INTERTIDAL HABITATS: WHAT IS THE POTENTIAL OF ECOLOGICAL ENGINEERING TO MITIGATE IMPACTS OF COASTAL STRUCTURES?
}

Matthew J. Perkins ${ }^{1,2}$, Terence P.T. $\mathrm{Ng}^{1,2}$, David Dudgeon ${ }^{1}$, Timothy C. Bonebrake ${ }^{l}$ and Kenneth M.Y. Leung ${ }^{1,2}$

${ }^{1}$ School of Biological Sciences, Faculty of Science, The University of Hong Kong, Pokfulam, Hong Kong SAR, China

${ }^{2}$ The Swire Institute of Marine Science, The University of Hong Kong, Hong Kong SAR, China

Globally, coastal human population growth and urbanization continues in a fast pace, while climatic change leads to stormier seas and rising tides. Both driving forces jointly generate a strong and increasing demand for land reclamation and infrastructure protection in coastal areas which require engineered defence structures such as sea walls. This study aimed to review ecological impacts of coastal engineered structures on intertidal ecosystems, update the current status of ecological engineering to mitigate these impacts, and evaluate the effectiveness of such mitigations in relation to the conservation of intertidal habitats and biodiversity. Engineered structures alter important physical, chemical and biological processes of intertidal habitats, and strongly impact community structure, inter-habitat linkages and ecosystem services while they can also drive habitat loss. Such impacts occur diffusely across localised sites but scale to significant regional and global levels. Promisingly, developments in ecological engineering have identified a need to increase habitat complexity on artificial structures. Soft engineering options maximise habitat complexity and sustain natural process while they can simultaneously deliver engineering objectives such as coastal protection. Soft options additionally sustain multiple services, providing greater economic benefits for society, and resilience to climatic change. Under-inclusion and economic undervaluation of intertidal ecosystem services may, however, undermine best practice in coastline management. Evidently, mitigation and even restoration through ecological engineering often do not support intertidal communities or processes that are equivalent to pre-disturbance conditions. Crucially though, an absence of comprehensive baseline biodiversity data, or data comprising additional ecological parameters such as ecosystem functions and services, prohibits quantification of absolute and relative magnitudes of ecological impacts due to engineered structures or effectiveness of mitigation interventions. This knowledge deficit restricts evaluation of the potential of ecological engineering to contribute to conservation policies for intertidal habitats. To improve mitigation design and effectiveness, a greater focus on in-situ research is needed, requiring stronger and timely collaboration between government agencies, construction engineers and research scientists. 